\title{
Chorea Huntington
}

\section{Der nicht alltägliche Fall bei „unklarer Bewusstseinsstörung"}

\author{
M. Philipp, Abteilung für Anästhesie und Intensivmedizin, Collm Klinik Oschatz, Oschatz
}

NOTFALLMEDIZIN 2003; 29: 416-418

Die im rettungsdienstlichen Alltag häufig anzutreffende ,unklare Bewusstseinsstörung" kann durch eine Chorea Huntington verursacht werden. Bei diesem Erbleiden kommt es zu einer fortschreitenden Funktionsstörung spezialisierter Hirnabschnitte, wobei der Erkrankungsbeginn zwischen dem 25. und 50. Lebensjahr liegen kann. Der betroffene Patient bietet eine Vielzahl neurologischer, vegetativer oder psychischer Auffälligkeiten, von welchen die motorische Unruhe und die unverkennbaren Hyperkinesien charakteristisch sind. Ein psychischer Abbauprozess führt frühzeitig zu Persönlichkeitsveränderungen mit Aggressivität bis hin zur paranoid-halluzinatorischen Psychose - Depressionen mit einer hohen Suizidrate stellen keine Seltenheit dar. Für eine mittels direktem Gennachweis erkannte Erkrankung steht bislang keine kausale Therapie zur Verfügung. Zur Antriebsminderung kann das hochpotente Neuroleptikum Haloperidol eingesetzt werden und stellt für den notfallmedizinischen Einsatz das Mittel der Wahl dar.

$\mathrm{D}$ er Rettungsdienst wird von der zuständigen Leitstelle auf Grund einer Nachforderung durch die ersteingetroffene Polizeistreife im Stadtgebiet einer Kleinstadt unter dem Notfallbild „hilflose Person“ zum Einsatz gebracht.

Beim zeitgleichen Eintreffen von Notarzt und Rettungswagen berichteten die Beamten, der Patient sei orientierungslos auf einer Hauptverkehrsstraße umhergelaufen, nachdem er sein Fahrzeug in einer Einfahrt noch abgestellt und verschlossen hatte. Ein Atemalkoholtest sei durchgeführt worden und negativ ausgefallen.

\section{Anamnese}

Im Erstbefund fand sich der 37jährige Patient in gutem Allgemeinzustand. Er reagierte anfangs eher verhalten, dann jedoch abweisend und aggressiv auf die Befragung durch den Notarzt. Eine Krankheitseinsicht bestand nicht, eine Behandlung wurde initial abgelehnt. Zu Zeitangaben und zu seiner Person war der Patient voll orientiert, örtlich jedoch nicht. Grob neurologisch stellte sich der Reflexstatus regel- recht dar - keine Einschränkung der Extremitätenmotorik, seitengleiche und mittelweite Pupillen mit normaler Lichtreaktion. Auffallend war ein deutlich linksseitig ausgeprägter Nystagmus. Bei der körperlichen Untersuchung fielen weiterhin ruckartige Kopf- und Extremitätenbewegungen - vornehmlich der Arme auf. Anhaltspunkte für den Gebrauch von Drogen, für eine andersartige Intoxikation, ein Anfallsleiden oder ein Schädel-Hirn-Trauma gab es nicht. Die einzige Medikation bestand in einer bedarfsweisen Applikation eines Antihistaminikums wegen einer Pollenallergie, welches jedoch nach Patientenangaben über Wochen nicht eingenommen wurde. Seitens der Vitalparameter stellte sich ein normofrequenter und mit einem systolischen Blutdruck von $140 \mathrm{mmHg}$ leicht hypertoner Kreislauf sowie eine unauffällige Atemphysiologie dar - $\mathrm{SaO}_{2}$-Werte von $98 \%$ bei Raumluft. Nach längerer Befragung berichtete der Patient von einer erblichen Chorea Huntington, die bei seinem Vater zum Tod geführt hatte. Eine Untersuchung sei bei ihm noch nie durchgeführt wor- den, er sei nach seiner Erinnerung bislang symptomfrei gewesen.

Dem Patienten wurde ein peripher-venöser Zugang gelegt und zur Sedierung 5 mg Haloperidol verabreicht. Die nunmehr erfolgte Kontrolle des Blutzuckerspiegels erbrachte einen normoglykämischen Wert. Unter Sedierung erfolgte die Einweisung des Patienten in eine neurologische Fachklinik mit der Arbeitsdiagnose „Verdacht des beginnendes Psychosyndroms bei Erstmanifestation einer Chorea Huntington“.

In der Notaufnahme erschien der Patient zwar bewusstseinsklarer und ausreichend orientiert, wirkte jedoch im Denkablauf noch leicht verworren. Es bestand weiter ein TIC-artiges Zucken des linken Augenlides und der Gesichts-Halsmuskulatur. Im weiteren neurologischen Befund fanden sich an pathologischen Veränderungen ein deutlich gesteigerter Muskeldehnungsreflex der oberen und unteren Extremitäten, ein beidseitig verlangsamter und hypometrischer Finger-NaseVersuch, eine beidseitige Dysdiadochokinese sowie ein ebenfalls beidseitig unsicherer Knie-HackeVersuch. Laborchemisch war nur eine Creatinkinase (CK) von $270 \mathrm{U} / 1$ (Normwert: < $171 \mathrm{U} / \mathrm{l}$ ) auffällig.

\section{Pathogenese}

Bei der Chorea Huntington (Huntington's Disease, HD), auch als Veitstanz (griechisch Chorea $=$ Tanz) bezeichnet, handelt es sich um eine Erkrankung des extrapyramidalen Systems. Dessen Aufgabe ist die Koordination von Bewegungsabläufen hinsichtlich Kraft, Belastung, Richtung, Geschwindigkeit und Amplitude. Im Jahr 1872 beschrieb George Huntington (Abb. 1) erstmals die 
Chorea major als erbliche Nervenerkrankung und grenzte sie somit von der Chorea minor, als Folge einer Streptokokkeninfektion, ab. Das autosomal-dominante Erbleiden mit fast vollständiger Penetranz (beide Geschlechter sind betroffen, die Kinder von Betroffenen haben ein 50\%iges Erkrankungsrisiko) gehört in gut erfassten Regionen mit einem Vorkommen von fast 10:100 000 Einwohnern zu den häufigsten neurologischen Erbkrankheiten $(5,6)$. Der Erkrankungsbeginn liegt zwischen dem 25. und dem 50. Lebensjahr. Nur 10\% aller Patienten erkranken früher - eine kindliche Erkrankungsmanifestation mit schwerem Verlauf und Überwiegen der betroffenen Mädchen ist möglich $(2,9)$.

Bereits 1983 wurde die HD auf dem kurzen Arm des Chromosoms 4 kartiert (4). Erst 1993 jedoch gelang der Nachweis des verantwortlichen, bisher nicht bekannten Gens, welches in vielen Geweben vorkommt. Bei Erkrankten erscheint in seinem kodierenden Abschnitt ein polymorphes Basentriplett (CAG - Cytosin/ Adenin/Guanin), wobei die Sequenz normalerweise nur 10-30 Tripletts enthält. Bei HD-Patienten kommen hier jedoch meist 40 und mehr Tripletts vor. Anhand der Anzahl der Tripletts kann auf das Manifestationsalter geschlossen werden - bei der niedrigsten Zahl an Tripletts von 37 muss die Erkrankung nicht unbedingt innerhalb der normalen Lebensspanne auftreten. Jedoch sinkt mit zunehmender CAG-Zahl das Manifestationsalter und bei über 60 CAG-Tripletts kann von einem kindlichen Auftreten ausgegangen werden (1). Interessant scheinen weiter die Erkenntnisse, dass etwa 3\% der Chorea-Huntington-Fälle Neumutationen sind und die Instabilität der Geninformation bei der Spermienentwicklung im Vergleich zur Entwicklung der Eizelle ausgeprägter ist. Der Krankheitsverlauf ist bei der Genübertragung durch den Vater beim Patienten meist schwerer $(3,10)$. Die genetische Beratung von Betroffenen ist somit möglich, wird jedoch nur unter psychologischem Beistand vorgenommen, da trotz Nachweis der Erkrankung eine kausale Therapie noch nicht zur Ver- fügung steht und sich mit Diagnosestellung einschneidende Folgen für den Patienten ergeben können.

Die klinische Symptomatik entsteht einerseits durch das Überwiegen der Wirkung des Dopamins (Neurotransmitter) durch Untergang von Ganglienzellen in spezifischen Hirnbereichen, nämlich im Nucleus caudatus und Nucleus lentiformis, und andererseits durch verminderte Wirkung von GABA (Gammaaminobuttersäure) im gesamten Corpus striatum und Globus pallidus. Nachgewiesen werden konnte ein Glukose-Hypometabolismus in Striatum und Kortex (8). Die Störung der mitochondrialen Atmungskette führt zu einem Energiedefizit, welches das Gehirn mit der Metabolisierung von Lactat auszugleichen versucht. Der trotz allem bestehende Energiemangel führt zu einer Empfindlichkeit gegenüber exzitatorischen Reizen, einem vermehrten Einstrom von Ca-Ionen mit weiterer Störung des Zellstoffwechsels und zur Bildung freier Radikale. Dies bewirkt mit der Funktionsstörung der Atmungskette vorerst eine Schädigung, dann jedoch das Absterben von Neuronen.

\section{Klinik}

Der Patient kann mit einer Vielzahl an Symptomen auffällig werden, welche sowohl neurologischen, vegetativen oder psychischen Charakters sein können. Kennzeichnend ist die dem choreatischen Syndrom zugrunde liegende motorische Unruhe. Die unverkennbaren Hyperkinesien werden durch unwillkürliche, blitzartig einsetzende und meist asymmetrisch-schleudernd ablaufende Kontraktionen einzelner Muskeln und Muskelgruppen hervorgerufen. Diese Erscheinungen sistieren im Schlaf und verstärken sich bei emotionaler Erregung (2). Aber auch hypokinetische Verlaufsformen mit entsprechend entgegengesetztem Verhalten sind beschrieben. Der Patient versucht, die nicht unterdrückbaren hyperkinetischen Erscheinungen vielfach in normale Bewegungsabläufe zu integrieren so wird etwa der nach oben schleudernde Arm in ein Streichen über die Stirn „verwandelt“. Der Muskeltonus ist beim Patienten dabei meist

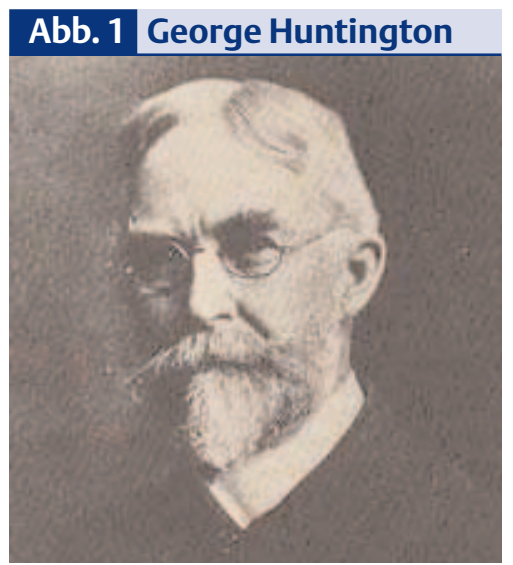

Beschrieb als Erster die Chorea major als erbliche Nervenerkrankung.

herabgesetzt. Die Sprachfähigkeit nimmt im Verlauf durch Artikulationsstörungen (Dysarthrie) in Lautgebung und Sprechablauf bis zur Unverständlichkeit ab. Häufig variiert die Lautstärke im Gespräch, oder es sind Schnalz- und Grunzlaute, Räuspern oder Schnaufen zu vernehmen. Auch die Mimik wird bald durch ein Grimassieren verstellt (9).

Vegetative Störungen sind gleichfalls stark variabel und äußern sich als Veränderungen des Appetits - im Endbild der Erkrankung sieht man häufig einen abgemagerten und vorzeitig gealterten Patienten -, der Libido, des Schlafes, der Thermoregulation mit Hang zur übermäßigen Schweißbildung (Hyperhidrosis) an den Akren, einer Akrozyanose oder Urininkontinenz $(2,9)$.

Vor Einsetzen dieser Symptome fällt jedoch meist ein psychisches Abbausyndrom auf, welches den neurologisch-vegetativen Störungen mitunter Jahrzehnte vorausgehen kann. Persönlichkeitsveränderungen mit Hang zur Aggressivität und Vernachlässigung bis hin zur paranoid-halluzinatorischen Psychose sind möglich. Das häufigste Frühsymptom ist die Depression mit einer hohen Suizidrate. Ein Zusammenleben mit dem Patienten ist aufgrund derartiger Veränderungen für die Familie meist schwierig. Besonders die Affektlabilität und die Antriebsschwäche stellen dabei vorrangige Probleme dar. Spätestens nach 10-15 Jahren ist bei allen Betroffenen eine Demenz nachzuweisen, die rein fokal beginnt und bei 


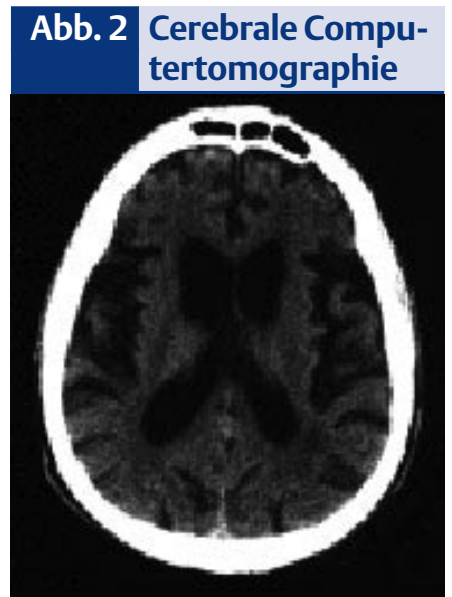

CCT mit Darstellung struktureller Veränderungen bei Huntington fortgeschrittener Hirnatrophie globale Defizite aufweist. Die frühzeitige Störung der Aufmerksamkeit und des Lernens führt ebenfalls recht früh zu Schwierigkeiten im beruflichen Alltag.

Hinweisgebend für die Diagnostik ist, neben der ausführlichen eigenund fremdanamnestischen Befragung, die klinische Untersuchung. Auffallend sind hierbei unter anderem das Phänomen der Chamäleonzunge - die Zunge kann vom Patienten nicht im herausgestreckten Zustand gehalten werden, sondern zuckt unwillkürlich zurück - und ein verzögertes Rückstellvermögen des Beines nach Auslösen des Patellarsehnenreflexes. Im Elektroenzephalogramm finden sich eher unspezifische Veränderungen, im Elektromyogramm bei Hyperkinesien typische Aktionspotentialausbrüche in den Agonisten, nicht jedoch bei den Antagonisten. An bildgebenden Verfahren hat sich zur Sicherung morphologischer Veränderungen die cerebrale Computertomographie (CCT) behauptet (Abb. 2). Dabei stellt sich zumeist eine Seitenventrikelerweiterung in Folge der Atrophie des Nucleus caudatus dar. Funktionell kann mit Hilfe der Positronen-Emissions-Tomographie (PET) der verminderte striale Glukosestoffwechsel zur Darstellung gebracht werden. Beweisend für das Vorliegen einer Chorea Huntington ist der verfügbare direkte Gennachweis mittels der Polymerase-Ketten-Reaktion (PCR).

\section{Therapie}

Für die Chorea Huntington steht keine kausale Therapie zur Verfügung. Eine neurologisch-psychologische Behandlung des Patienten und der Angehörigen wird angestrebt. Einzig die Hyperkinese kann medikamentös wirksam mit Hilfe von $\mathrm{D}_{2}{ }^{-}$ Antagonisten, welche die Wirksam- keit des Dopamins herabsetzen, angegangen werden. Die damit gegebenenfalls verbundene Sedierung kann bei Reizbarkeit und Affektlabilität wünschenswert sein. Eine mögliche Behandlung mit GABA-ergen Substanzen, zum Ausgleich des Verhältnisses Dopamin-GABA, wird wegen ernster Nebenwirkungen nur äußerst selten verwendet. Zur Antriebsminderung hat sich die Gabe des hochpotenten Neuroleptikums Haloperidol bewährt und kann auch beim Notfallpatienten mit Verdacht der Chorea Huntington - unter Beachtung der Kontraindikationen problemlos eingesetzt werden. Haloperidol aus der Gruppe der Butyrophenone zeichnet sich durch eine stark antipsychotische Wirkung aus, gepaart mit nur geringer Sedierung und kaum vorhandener anticholinerger Eigenschaft (7). Es ist daher präklinisch Mittel der Wahl. Die Medikation mit Radikalfängern, Ca-Antagonisten und verschiedenen Vitaminen bleibt der Dauertherapie vorbehalten und ist teils noch von experimentellem Charakter.

Die im Rettungsdienst vielfach anzutreffende „unklare Bewusstseinsstörung“ kann durchaus auf Grundlage der Chorea Huntington auftreten. Die Wahrscheinlichkeit mit einem solchen Patienten präklinisch konfrontiert zu werden, ist angesichts der im notfallmedizinischen Vergleich doch geringeren Prävalenz als nicht hoch einzustufen. Gerade deswegen sollen jedoch die Ausführungen dazu beitragen, neben allen häufigen Gründen der Bewusstseinstörungen - wie Traumata und Intoxikationen - auch an diese Ursache mitzudenken. Denn die Grenzbereiche des alltäglichen Notfalls stellen immer wieder Herausforderungen an alle im Rettungsdienst Tätigen dar - ob Rettungssanitäter oder Notarzt.

\section{Summary}

The patient with unclear clouding of consciousness, as is commonly encountered in the daily routine of emergency medical services, may be due to Huntington's chorea. During the course of this hereditary disease, progressive functional disordering of specific parts of the brain occurs, the onset of the disease lying between age 25 and 50 years. The affected patient presents with numerous neurological, vegetative or psychiatric abnormalities, of which motoric superactivity and unmistakable hyperkinesias are typical. Mental deterioration early leads to changes in personality accompanied by aggression that may progress to a paranoid-hallucinatory psychosis; progressive states associated with a higher suicide rate are not infrequent. For a genetically-based diagnosed of this disease we currently have no causal treatment available. As a means of reducing drive, the highly potent neuroleptic agent, haloperidol can be administered, and this is the drug of choice for use in an emergency.

\section{Literatur}

1. Duayo M, Ambrose C, Myers R et al. Trinucleotide repeat length and age of onset in Huntington's disease. Nature Genetica 1993; 4: 387-392

2. Gleixner C, Müller M, Wirth S. Neurologie und Psychiatrie. Breisach: Medizinische Verlags- und Informationsdienste, 1. Auflage $1998 / 1999$

3. Goldberg YP, Kremer B, Andrew SE et al. Molecular analysis of new mutations for Huntington's disease: intermediate alleles and sex of origin effects. Nature Genetics 1993; 5: 174-179

4. Gusella JF, Wexler NS, Conneally PM et al. A polymorphic DNA marker genetically linked to Huntington's disease. Nature 1983; 306: 234-238

5. Harper PS. Huntington's disease. London: Saunders, 1991

6 . Hayden MR Huntington's chorea. Berlin: Springer, 1981

7. Karow T, Lang R. Allgemeine und spezielle Pharmakologie und Toxikologie. Pulheim: Karow, 1999

8. Quadbeck H, Lange HW, Wieler H, Feinendegen LE. The cerebral oxidative glucosemetabolism in patients with Huntington's chores. Abstr., World Congress of Biol. Psychiatry, Philadelphia, Sept. 8.-13.1985 9. Scheid W, Gibbels E. Lehrbuch der Neurologie. Stuttgart-New York: Georg Thieme Verlag, 5. Auflage, 1983

10. Zühlke C, Reiß O, Bockel B et al. (1993) Mitotic stability and meiotic variability of the (CAG)n reapeat in the Huntington's disease gene. Human Molecular Genetics 1993; 2: 2063-2067

\section{Anschrift des Verfassers Mike Philipp c/o Collm Klinik Oschatz Abteilung für Anästhesie und Intensivmedizin \\ Parkstraße 1 \\ 04758 Oschatz}

